\title{
The Majumdar-Ghosh chain. Twofold ground state and elementary excitations
}

\author{
W J Caspers $\nmid$, K M Emmett $\dagger$ and W Magnus $\ddagger$ \\ $\dagger$ Center of Theoretical Physics, Twente University of Technology, Enschede, The \\ Netherlands \\ $\ddagger$ Instituut voor Theoretische Fysika, University of Leuven, B-3030 Leuven, Belgium
}

Received 9 Ápril 1984

Abstract. Recently it was proved that the Majumdar-Ghosh chain with the Hamiltonian

$$
H=4 \sum_{j=1}^{2 N} S_{j} \cdot S_{j+1}+2 \sum_{j=1}^{2 N} S_{j} \cdot S_{j+2}, \quad S_{\imath+2 N} \equiv S_{\imath}, \quad S_{i}=\frac{1}{2}
$$

has at least two ground states, in which the spins are arranged in nearest-neighbour singlet pairs. In this work it is shown that these two states are the only ground states. Besides, a rapidly converging variational method is given to determine the elementary excitations.

\section{Introduction}

This work is about the ground states and elementary excitations of the linear spin system introduced by Majumdar and Ghosh (1969a, b) (MG). The MG chain is, in our restricted definition, a linear system of spins $\frac{1}{2}$ with an isotropic interaction (exchange) between nearest and next-nearest neighbours, and the interaction constant for nextnearest neighbours is $\frac{1}{2}$ of that for nearest neighbours.

It was suggested already in Majumdar and Ghosh (1969b) that the system has at least two ground states, the singlet-pair states (SPS), in which the spins are arranged in nearest-neighbour singlet pairs. The two sps are asymptotically $(N \rightarrow \infty)$ orthogonal and are connected by a translation over one elementary distance.

Van den Broek (1980) gave the proof that the two SPS are indeed ground states for arbitrary $N$. So far it has remained an open question whether or not these two states are the only ground states.

An analogous proof for the existence of a ferromagnetic ground state for a chain with competing interactions between neighbours and next-nearest neighbours has been given by Bader and Schilling (1979).

In $\S 2$ it is proved that the two sPS are the only ground states for the system with periodic boundary conditions, the closed MG chain. For the open chain there is only one ground state.

The MG chain confronts us also with the following interesting question. Is there a lower bound in the energy spectrum of the elementary excitations (Majumdar 1970, Shastry and Sutherland 1981, Caspers and Magnus 1982, 1983a)? Here we follow the suggestion of Shastry and Sutherland (1981), who introduced excitations consisting of the two sps 'phases', separated by a single 'defect'. Even-numbered chains necessarily 
contain an even number of these defects and in Shastry and Sutherland (1981), by means of a variational calculation, an upper bound for the lowest possible energy for two of these excitations was determined, in bound and unbound states.

Section 3 of this paper is devoted to the analysis, in somewhat more detail, of excitations consisting of one single 'defect' in an odd-numbered chain. Starting with Shastry and Sutherland's suggestion of one single spin, separating two SPS phases, we construct a variational wavefunction that contains also other components. Our calculations result in a minimum energy per free defect that is somewhat lower than the value of Shastry and Sutherland (1981).

Combination of two of these defects may result in scattering states, the energy of which is simply the sum of the energies of the two defects, or in bound states. Two exact excited states already known (Majumdar 1970, Caspers and Magnus 1982) may be considered as bound states of these defects.

Two unbound defects with minimum energy result in the lowest excitation energy that was found till now. The corresponding 'gap' in the energy spectrum is consistent with numerical results for small systems (Majumdar et al 1972).

In $\S 4$ the possibility of a twofold ground state, also of the sps type, is discussed for the generalisations of the MG chain (Caspers 1982, Caspers and Magnus 1983b). In this context we may also draw attention to the work of Klein (1982), who also discussed two-dimensional generalisations of the SPS.

\section{Twofold ground state}

According to van den Broek (1980), Caspers (1982) and Caspers and Magnus (1983b) the Hamiltonian of the MG chain with periodic boundary conditions may be written

$$
\begin{aligned}
H=4 \sum_{j=1}^{2 N} \boldsymbol{S}_{j} \cdot \boldsymbol{S}_{j+1}+2 \sum_{j=1}^{2 N} \boldsymbol{S}_{j} \cdot \boldsymbol{S}_{j+2} \\
=2 \sum_{j=1}^{2 N}\left(\boldsymbol{S}_{j} \cdot \boldsymbol{S}_{j+1}+\boldsymbol{S}_{j+1} \cdot \boldsymbol{S}_{j+2}+\boldsymbol{S}_{j} \cdot \boldsymbol{S}_{j+2}\right)=\sum_{j=1}^{2 N} \bar{H}_{j}, \quad \boldsymbol{S}_{i+2 N} \equiv \boldsymbol{S}_{i},
\end{aligned}
$$

with

$$
\bar{H}_{j}=\left(\boldsymbol{S}_{j}+\boldsymbol{S}_{j+1}+\boldsymbol{S}_{j+2}\right)^{2}-\frac{9}{4} .
$$

The two sps are eigenstates of $H$ with lowest possible eigenvalue because they are eigenstates of all individual $\bar{H}_{j}$ with corresponding lowest eigenvalue $\frac{3}{4}-\frac{9}{4}=-\frac{3}{2}$.

The most important property of the sPs is that the total spin of all triples $(j, j+1, j+$ 2 ) is $\frac{1}{2}$. This property necessarily holds for all possible ground states of $H$, because all $\bar{H}_{j}$ should have their lowest eigenvalue $-\frac{3}{2}$. It is our aim to prove that this is only fulfilled for the two sPs.

The first step in the argument is to write all possible configurations of three consecutive spins in terms of Ising states, i.e. eigenstates of the individual $S_{i z}, i=$ $1,2, \ldots, 2 N$. In general there exist eight three-spin states:

$$
\begin{array}{llll}
\left|\psi_{1}\right\rangle=|++-\rangle, & & \left|\psi_{4}\right\rangle=|--+\rangle, & \\
\left|\psi_{2}\right\rangle=|+-+\rangle, & & \left|\psi_{5}\right\rangle=|-+-\rangle, & \left|\psi_{7}\right\rangle=|+++\rangle, \\
\left|\psi_{3}\right\rangle=|-++\rangle, & & \left|\psi_{6}\right\rangle=|+--\rangle, & \left|\psi_{8}\right\rangle=|---\rangle .
\end{array}
$$


The three positions in the kets correspond e.g. with the spins $j, j+1, j+2$ respectively, a $+(-)$ sign denoting an eigenstate of an $S_{i z}(i=j, j+1, j+2)$ with eigenvalue $+\frac{1}{2}\left(-\frac{1}{2}\right)$. As was shown before in the ground state of the chain only three-spin states corresponding with total spin $\frac{1}{2}$ should appear. So we should exclude $\left|\psi_{7}\right\rangle$ and $\left|\psi_{8}\right\rangle$ in a possible construction of a ground state of the chain. The only configurations that are allowed are the following:

$$
|\psi\rangle=\sum_{n=1}^{6} a_{n}\left|\psi_{n}\right\rangle, \quad a_{1}+a_{2}+a_{3}=0, \quad a_{4}+a_{5}+a_{6}=0,
$$

corresponding with the four-dimensional eigenspace of the restriction of $\bar{H}_{j}$, for the eigenvalue $-\frac{3}{2}$. So we may conclude that in the Ising states that appear in the ground state of the chain there are no more than two + or - spins in a row, and the allowed configurations of three consecutive spins obey (4).

In the next step we may draw the conclusion that all relevant Ising states for the chain are fully characterised by the position of the ++ and - neighbour pairs, with the exception of the two Néel states:

$$
|+-+-+-+-\ldots+-\rangle, \quad|-+-+-+-+\ldots-+\rangle .
$$

Now we will prove that configurations in which a ++ pair is followed by another ++ pair, wherever located, have amplitude zero, and in order to do so we consider the following Ising states:

$$
\begin{aligned}
& |\ldots++-+-+-+\ldots+-+-++\ldots\rangle, \\
& |\ldots++-+-+-+\ldots+-++-+\ldots\rangle, \\
& |\ldots++-+-+-+\ldots++-+-+\ldots\rangle, \\
& |\ldots++-++-+-\ldots-+-+-+\ldots\rangle,
\end{aligned}
$$

in which in all kets the configurations indicated by the dots are the same, with the restrictions already formulated. The configuration between the two ++ pairs is the Néel state in all kets. The last ket makes an exception to these rules: the Néel state indicated by the middle group of dots is the time inversion of the corresponding state in the other kets.

Now it is easily proved with the aid of (4) that, if the amplitude of the first ket is $x$, the amplitude of the second is $-x$, of the third $x$, etc. The last one necessarily has an amplitude $\pm x$, but considering the configuration ++-++ at the front end, one easily proves that $x=0$, on the basis of (4) applied on the second, third and fourth spin of this group of five. In the same way one may state that a -- pair can never be followed by a - - pair.

In an analogous way we may consider a set of states in which a $++(--)$ pair is followed by a - $(++)$ pair, and instead of (6) we now have

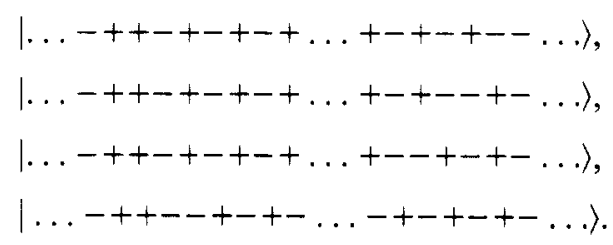

Again, in between the two equal pairs we have a Néel configuration. In an analogous 
way as was done for the set (6) we may prove that the amplitudes of the set (7) should be $x,-x, x, \ldots, \pm x$. But now the last one does not necessarily have an amplitude 0 , so $x$ may be unequal to 0 in this case. Considering the triple consisting of the second, third and fourth spin of the first group of four spins, in the last ket, which has an amplitude $\pm x$, one easily sees that this amplitude is connected by (4) with the amplitudes of

and

$$
|\ldots-+-+-+-+-\ldots-+-+-+\ldots\rangle
$$

$$
|\ldots--++-+-+-\ldots-+-+-+\ldots\rangle \text {. }
$$

The last ket in (8) only exists if in the states in (7) there is only one ++ and one - pair, otherwise there would be a - - pair followed by another - - pair in this last ket, and that was already excluded.

Now it is convenient to introduce an index to define the position of the equal pairs. The index $j$ introduced in (1) defines the position of the single spins in the chain $1 \leqslant j \leqslant 2 N$, and an index $n$ may be used to fix the positions $(2 n-1,2 n)$ or $(2 n, 2 n+1)$ of equal pairs. In this way one may distinguish six types of states:

$$
\begin{aligned}
& \left.\Phi_{+}\left(n_{1}, n_{2}, n_{3}, \ldots, n_{2 P}\right),++ \text { at positions }\left(2 n_{2 k-1}-1,2 n_{2 k-1}\right)\right) \\
& \text { - at positions }\left(2 n_{2 k}-1,2 n_{2 k}\right) \\
& \begin{array}{r|l}
\Phi_{-}\left(n_{1}, n_{2}, n_{3}, \ldots, n_{2 P}\right),++ \text { at positions }\left(2 n_{2 k}-1,2 n_{2 k}\right) & \begin{array}{l}
k=1,2,3, \ldots, P, \\
1 \leqslant n_{1}<n_{2}<\ldots<n_{2 P} \leqslant N,
\end{array}
\end{array} \\
& \left.\Psi_{+}\left(n_{1}, n_{2}, n_{3}, \ldots, n_{2 P}\right),++ \text { at positions }\left(2 n_{2 k-1}, 2 n_{2 k-1}+1\right)\right\} P=1,2, \ldots,[N / 2] \text {, } \\
& \text { - at positions }\left(2 n_{2 k}, 2 n_{2 k}+1\right) \quad(2 N, 2 N+1) \equiv(1,2 N), \\
& \Psi_{-}\left(n_{1}, n_{2}, n_{3}, \ldots, n_{2 P}\right),++ \text { at positions }\left(2 n_{2 k}, 2 n_{2 k}+1\right) \\
& -- \text { at positions }\left(2 n_{2 k-1}, 2 n_{2 k-1}+1\right) \\
& \chi_{+}=|+-+-+-\ldots+-\rangle, \quad \chi_{-}=|-+-+-+\ldots-+\rangle .
\end{aligned}
$$

The two states $\chi_{ \pm}$are the two possible Néel states for the complete chain.

The analysis that resulted in the selection of the six types of states shown in (9) also gives us a simple relation for the amplitude of all $\Phi_{+}$for given $P$; the same is true for the $\Phi_{-}, \Psi_{+}$and $\Psi_{-}$. These amplitudes are respectively given by table 1 .

Table 1. Amplitudes of Ising states.

\begin{tabular}{lll}
\hline$\Phi_{+}\left(n_{1}, n_{2}, \ldots, n_{2 P}\right)$ & $x_{P}(+) F\left(n_{1}, n_{2}, \ldots, n_{2 P}\right)$ \\
$\Phi_{-}\left(n_{1}, n_{2}, \ldots, n_{2 P}\right)$ & $x_{P}(-) F\left(n_{1}, n_{2}, \ldots, n_{2 P}\right)$ \\
$\Psi_{+}\left(n_{1}, n_{2}, \ldots, n_{2 P}\right)$ & $y_{P}(+) F\left(n_{1}, n_{2}, \ldots, n_{2 P}\right)$ & \\
$\Psi_{-}\left(n_{1}, n_{2}, \ldots, n_{2 P}\right)$ & $y_{P}(-) F\left(n_{1}, n_{2}, \ldots, n_{2 P}\right)=\left(-n^{n_{1}+n_{2}} \quad+n_{2 P}\right.$ \\
\hline
\end{tabular}

In comparing the amplitudes of the states given in (7) and (8) with the restriction that the states in (7) contain more than one pair of both types ++ and -- , one immediately derives that the $x_{P}(+)$ etc are independent of $P: x_{P}(+)=x(+), x_{P}(-)=$ $x(-), y_{P}(+)=y(+), y_{P}(-)=y(-)$. On the basis of the periodic boundary conditions 
imposed on the system one derives that the sum of the amplitudes of the following states is zero:

$\Phi_{+}\left(n_{1}=1, n_{2}, n_{3}, \ldots, n_{2 P-1}, n_{2 P}=N\right), \quad \Phi_{-}\left(n_{2}, n_{3}, \ldots, n_{2 P-1}\right), \quad P \geqslant 2$,

from which it immediately follows that $x(+)=(-)^{N} x(-)=x$. In an analogous way it holds that $y(+)=(-)^{N} y(-)=y$.

Finally the two parameters $x$ and $y$ determine the amplitude of the two Néel states $\chi_{+}$and $\chi_{-}$. The sum of the amplitudes of the state $\chi_{+}$and the states that start with the configurations ++--+ and +--++ respectively and are otherwise identical with $\chi_{+}$equals zero. Consequently the amplitude of $\chi_{+}$equals $x+(-)^{N} y$, and in the same way one finds the amplitude of $\chi$ - to be $(-)^{N} x+y$.

The two possible sPs may be written

$$
\begin{aligned}
& \left|\Phi_{0}\right\rangle=|0\rangle_{1,2}|0\rangle_{3,4}|0\rangle_{5,6} \ldots|0\rangle_{2 N-1,2 N}, \\
& \left|\Psi_{0}\right\rangle=|0\rangle_{2,3}|0\rangle_{4,5}|0\rangle_{6,7} \ldots|0\rangle_{2 N, 2 N+1} .
\end{aligned}
$$

As was proved in this section, all ground states of the Hamiltonian (1) may be expressed in terms of two independent parameters $x$ and $y$, and consequently all linear combinations of $\left|\Phi_{0}\right\rangle$ and $\left|\Psi_{0}\right\rangle: \xi\left|\Phi_{0}\right\rangle+\eta\left|\Psi_{0}\right\rangle$ may also be determined by the set $(x, y)$.

Considering the Ising states starting with the configurations ++--+ and +--++ and having a Néel ordering for the rest of the chain, and realising that the first one is a component of $\left|\Psi_{0}\right\rangle$ and the second of $\left|\Phi_{0}\right\rangle$, one finds

$$
\xi=(-)^{N} y, \quad \eta=(-)^{N} x .
$$

In this way we have completed the proof that the two SPS are the only ground states and have given a relation between these two states and the set constructed in this section. This is for a system with periodic boundary conditions.

For open ends, also for an even number of spins, the Hamiltonian $H$ may be written

$$
H^{\prime}=4 \sum_{j=1}^{N-1} S_{j} \cdot S_{j+1}+2 \sum_{j=1}^{N-2} S_{j} \cdot S_{j+2}=\sum_{j=1}^{2 N-2} \bar{H}_{j}+2 S_{1} \cdot S_{2}+2 S_{2 N-1} \cdot S_{2 N} \text {. }
$$

For this Hamiltonian one easily proves that again $\left|\Phi_{0}\right\rangle$ is a ground state, which is not the case for $\left|\Psi_{0}\right\rangle$. In an analysis completely analogous to the one given before, one proves that all ground states of $H^{\prime}$ are also ground states of $\bar{H}_{j}(j=1, \ldots, 2 N-2)$, $2 S_{1} \cdot S_{2}$ and $2 S_{2 N-1} \cdot S_{2 N}$. This leads to a set of Ising states given in (9) with the exclusion of $\Phi_{+}$and $\Phi_{-}$, consequently $x=0$, which completes the proof.

\section{Elementary excitations}

Shastry and Sutherland (1981) considered elementary excitations that consisted of a single unpaired spin, separating two different SPS phases, shifted with respect to another over one elementary distance. In the even-numbered chains the number of these excitations or defects should always be even.

These states with a fixed even number of defects, however, are not eigenstates of Hamiltonian (1), and the same is true for linear combinations which represent defects with wavenumbers $k$. But these latter states are variational for the correct excited states and Shastry and Sutherland (1981) determined along these lines scattering and bound states of two defects. 
In this paper we consider, in the first instance, only free defects and suppose that they can always be described by two asymptotic regions corresponding with the two sps phases, connected by a 'stacking' fault of finite size. In this picture it is immediately clear that for scattering states their energy is also additive. The description of the 'stacking' fault can be made more sophisticated than in the Shastry and Sutherland model by adding to the variational wavefunction other terms, which are generated by the Hamiltonian acting on the simple defect state. These extra terms correspond with five or nine consecutive spins separating two sps phases.

From numerical results it follows quite clearly that the procedure is rapidly converging, at least if one restricts oneself to three terms only: the last term is very small whereas the Shastry and Sutherland term is predominant.

A system in which there exists only one defect necessarily contains an odd number of spins, which we choose to be $P \equiv 2 N+1$. Our Hamiltonian is again of the form (1) with the number $2 N$ replaced by $P$. The zero-order expression for a defect state now reads:

$$
|\psi\rangle_{j}=|0\rangle_{1,2}|0\rangle_{3,4} \ldots|0\rangle_{j-2, j-1}|+\rangle_{j}|0\rangle_{j+1, j+2} \ldots|0\rangle_{2 N, 2 N+1} .
$$

The symbol $|0\rangle_{i, i+1}$ denotes a SPS for the pair $(i, i+1)$ and the defect is located at site $j$ and has spin up. The effect of the Hamiltonian $H$ on $|\psi\rangle_{j}$ is threefold:

(i) it reproduces $|\psi\rangle$,

(ii) it generates states $|\psi\rangle_{j-2}$ and $|\psi\rangle_{j+2}$,

(iii) it generates a state $|a\rangle_{j}$.

The defect may jump over two elementary distances in both directions, resulting in $|\psi\rangle_{j \pm 2}$. But also a state with a more complicated defect may result, the state $|a\rangle_{j}$, which corresponds with an SPS for the pairs $(1,2),(3,4), \ldots,(j-4, j-3), \ldots,(j+3, j+$ $4), \ldots,(2 N, 2 N+1)$ and a state with $S=\frac{1}{2}, M=\frac{1}{2}$ for the spins $(j-2, j-1, j, j+1, j+2)$ orthogonal to $|\psi\rangle_{j-2},|\psi\rangle_{j}$ and $|\psi\rangle_{j+2} .(S, M)$ represent total spin and $z$ component for the defect.

A straightforward calculation of the relevant part of $|a\rangle_{j}$ gives

$$
\begin{gathered}
\| a\rangle_{j}=(1 / 2 \sqrt{3})[2|+++--\rangle+2|--+++\rangle-|+-+-+\rangle-|-+++-\rangle \\
-1+-++-\rangle-|-++-+\rangle]_{(j-2, \ldots, j+2),}
\end{gathered}
$$

a normalised ket for the spins at position $(j-2, j-1, j, j+1, j+2)$. The state $|a\rangle_{j}$ is found by multiplying this ket with a state in which all the other spins are ordered in singlet neighbour pairs.

It should be stressed that the states $|\psi\rangle_{j}|\psi\rangle_{j \pm 2}$ are non-orthogonal. This procedure of generating defect states may be repeated, resulting in a component of $H^{2}|\psi\rangle_{j}$ (or $H|a\rangle_{j}$ ) orthogonal to $|\psi\rangle_{j},|\psi\rangle_{j \pm 2},|\psi\rangle_{j \pm 4},|a\rangle_{j},|a\rangle_{j \pm 2}$, which will be called, in its normalised form, $|e\rangle_{j}$. This defect state contains a relevant part $\left.\| e\right\rangle_{j}$ (cf $\left.\| a\right\rangle_{j}$ given in (14)) that can be found in the appendix.

So we finally propose a variational defect state, which is a linear combination of the states $|\psi\rangle_{j},|a\rangle_{j}$ and $|e\rangle_{j}$ for different $j$, and which belongs to a given representation of the translation group of the chain. The selection rules for $H$, however, imply that states with even (odd) index $j$ are only connected with states with even (odd) index $j$ ! Consequently only even (odd) indices $j$ have to be included in the Fourier sum for given $k$. For periodic boundary conditions, however, the spin defect at the odd position $2 N+1$, which in its most simple form is described by

$$
|\psi\rangle_{2 N+1}=|0\rangle_{1,2}|0\rangle_{3,1} \ldots|0\rangle_{2 N-1,2 N}|+\rangle_{2 N+1},
$$


may perform a transition to

$$
|\psi\rangle_{2}=|0\rangle_{2 N+1,1}|+\rangle_{2}|0\rangle_{3,4}|0\rangle_{2 N-1,2 N}
$$

so all $2 N+1$ positions are available for the spin defect. Effectively we have periodic boundary conditions for the defect states, corresponding with a system of length $2(2 N+1)$ times the elementary distance. The variational defect state will take the form

$$
\left|\Psi_{\mathrm{T}}\right\rangle=\sum_{\substack{j=2 \\(j \text { even })}}^{4 N+2} \mathrm{e}^{i k j}\left[a|\psi\rangle_{j}+b|a\rangle_{j}+c|e\rangle_{j}\right], \quad|\psi\rangle_{2 N+1+j} \equiv|\psi\rangle_{j} \text { etc, }
$$

and we intend to calculate the expectation value of $H$ for this generally not normalised state:

$$
E_{\mathrm{T}}=\langle H\rangle=\left\langle\Psi_{\mathrm{T}}|H| \Psi_{\mathrm{T}}\right\rangle /\left\langle\Psi_{\mathrm{T}} \mid \Psi_{\mathrm{T}}\right\rangle .
$$

The minimum of $\langle H\rangle$ as a function of $k$ will give us the best trial function for our defect. To determine (18) we first calculate all the possible inner products of the normalised kets that appear in (17). The results are given in table 2:

Table 2. Inner products of the kets $|\psi\rangle_{j},|a\rangle_{j}$ and $|e\rangle_{r}$

$\begin{array}{rlrl}j^{\prime}\langle\psi \mid \psi\rangle_{j} & =\left(-\frac{1}{2}\right)^{\mid j-j^{\prime} / 2} & & j, j^{\prime} \text { even, }\left|j-j^{\prime}\right| \leqslant 2 N \\ & =\left(-\frac{1}{2}\right)^{2 N+1-\mid j-j^{\prime} / 2} & & j, j^{\prime} \text { even, }\left|j-j^{\prime}\right| \geqslant 2 N+2 \\ j^{\prime}\langle a \mid a\rangle_{j} & =\delta_{j^{\prime} j} & & \\ j^{\prime}\langle e \mid e\rangle_{j} & =\delta_{j^{\prime} j}+\frac{1}{4}\left(\delta_{j^{\prime} j+2}+\delta_{j^{\prime} j-2}\right) & \\ j^{\prime}\langle a \mid \psi\rangle_{j} & =j_{j}\langle e \mid \psi\rangle_{j}={ }_{j^{\prime}}\langle a \mid e\rangle_{j}=0 & \end{array}$

Secondly we give, in table 3 , the effect of the Hamiltonian $H$ on the states $|\psi\rangle_{j},|a\rangle_{j}$ and $|e\rangle_{j}$ :

Table 3. Effect of $H$ on $|\psi\rangle_{j}|a\rangle_{\text {, and }}|e\rangle_{j}$

$$
\begin{aligned}
& H|\psi\rangle_{j}=(-3 N+1)|\psi\rangle_{j}+|\psi\rangle_{j-2}+|\psi\rangle_{j+2}+\frac{1}{2} \sqrt{3}|a\rangle_{j} \\
& H|a\rangle_{j}=(-3 N+7)|a\rangle_{j}+\frac{5}{6} \sqrt{3}|\psi\rangle_{j}+\frac{1}{3} \sqrt{3}\left(|\psi\rangle_{j-2}+|\psi\rangle_{j+2}\right)+2|e\rangle_{j} \\
& H|e\rangle_{j}=\left(-3 N+\frac{19}{2}\right)|e\rangle_{j}+2|a\rangle_{j}+4|g\rangle_{j}
\end{aligned}
$$

The states $|g\rangle_{j}$ are orthogonal to all $|\psi\rangle_{j^{\prime}},|a\rangle_{j^{\prime}}$ and $|e\rangle_{j^{\prime}}$ for arbitrary $j$ and $j^{\prime}$. For the case when we take $c=0$ in the variational state (17), we find for the value of (18)

$$
\begin{aligned}
& E_{\mathrm{T}}=-3 N+\frac{-\frac{3}{2} \kappa|a|^{2}+\frac{1}{2} \sqrt{3}\left(a^{*} b+a b^{*}\right)+7|b|^{2}}{|a|^{2}(\kappa+1)+|b|^{2}} \equiv-3 N+\Delta E_{\mathrm{T}}, \\
& \kappa=\sum_{s=1}^{\infty}\left(-\frac{1}{2}\right)^{s} 2 \cos 2 k s,
\end{aligned}
$$

in which the value for $\kappa$ is asymptotically correct for large $N$. From (19) it is immediately clear that taking $z=b / a=r \mathrm{e}^{\mathrm{i} \phi}$, extrema of $E_{\mathrm{T}}\left(\Delta E_{\mathrm{T}}\right)$ as a function of $\phi$ are reached 
for $\phi=0$ or $\pi$. We find the following values for these extrema:

$$
\Delta E_{\mathrm{T}}=\left(-\frac{3}{2} \kappa \pm \sqrt{3} r+7 r^{2}\right) /\left(\kappa+1+r^{2}\right), \quad \cos \phi= \pm 1 .
$$

First we have determined the minimum of $\Delta E_{\mathrm{T}}$ as a function of $r$, for fixed $\kappa$. Doing so we find that the minimum is reached for

$$
r=r_{\min }=\frac{+\left[(17 \kappa+14)^{2}+12(\kappa+1)\right]^{1 / 2}-(17 \kappa+14)}{2 \sqrt{3}}, \quad \phi=\pi .
$$

Substituting these results in (20) gives us a $\Delta E_{\mathrm{T}}$ that is a function of $\kappa$ only, and this function reaches its minimum at the boundary value $\kappa=2\left(k= \pm \frac{1}{2} \pi\right)$ :

$$
\Delta E_{\mathrm{T}}^{(1)}\left(r_{\min }(\kappa=2)\right)=-1.03113 \text {. }
$$

The more accurate calculations in which the terms $|e\rangle_{j}$ are also included in the variational state (17) lead to a final result that is only slightly different from (22):

$$
\Delta E_{\mathrm{T}}^{(2)}=-1.03196 \text {. }
$$

Details of the very lengthy calculations in which use was made of the method of Lagrange undetermined multipliers, which were performed with a series of Fortran programs, are omitted here. The values (22) and (23) suggest, however, that our method leads to rapidly converging results. Inclusion of more terms in the series (17) does not seem relevant.

These values should be compared with results in the literature which refer to even-numbered chains. An even-numbered chain may be subdivided in two parts with numbers $2 N_{1}+1$ and $2 N_{2}+1$, and we may take $N_{1}$ and $N_{2} \gg 1$. In these two parts subject to the same boundary conditions as above, for each part separately a wavepacket can be constructed by taking wavefunctions with a range $\Delta k_{1}, \Delta k_{2}$ of the respective wavevectors $k_{1}, k_{2}$. The $\Delta k$ are chosen large enough to localise the wavepacket within the boundaries of each part of the chain, but not so large that the energy becomes undefined; asymptotically the energy of this system will differ negligibly from the treatment given above. For the excited states considered in this section the lowest energy therefore equals

$$
-3\left(N_{1}+N_{2}\right)+2 \Delta E_{\mathrm{T}}=-3\left(N_{1}+N_{2}+1\right)+\left(2 \Delta E_{\mathrm{T}}+3\right)=E_{0}+\left(2 \Delta E_{\mathrm{T}}+3\right)
$$

in which $E_{0}=-3\left(N_{1}+N_{2}+1\right)$ is simply the ground state energy. So the elementary excitations have a lower bound for their energy, which equals

$$
\varepsilon=2 \Delta E_{\mathrm{T}}+3 \text {, }
$$

which for the best approximation (23) has the value

$$
\varepsilon\left( \pm \frac{1}{2} \pi\right)=0.93608 \text {. }
$$

The result of Shastry and Sutherland (1981), which corresponds with $a=1, b=0$ in formula (19), equals 1 . In their expression for the energy $\varepsilon$,

$$
\varepsilon=J\left(\frac{5}{2}-2|\cos Q|\right) \text {, }
$$

one should take $Q=0, \pi$ and $J=2$.

Majumdar et al (1972) performed calculations for short chains of 6, 8, 10 and 12 spins, and reported on their results in a graphical way, from which it could be inferred that their lowest excitation also corresponds with $\varepsilon \approx 1$. 
There already exist in the literature exact values for the energy of excitations of a special type for even-numbered chains of an unlimited number of spins (Caspers and Magnus 1982, Majumdar et al 1972). These excitations correspond with states in which one or two singlet pairs are decoupled and put in a triplet state. One single triplet pair represents an energy 4 , and the corresponding excitation energies are 4 and 8 . Two of these excitations, however, may form a bound state, in which an energy 4 is regained. We originally had the intention to describe all possible excitations in the MG chain with these triplet pairs, but came to the conclusion that one does not find the lowest excited states along these lines, at least not in an easy way.

We now firmly believe that (25) represents the best approximation up till now for the first excited state of the MG chain, but a rigorous proof that the energy spectrum has a gap above the ground state has not been given so far.

\section{Ground states for generalisations of the Majumdar-Ghosh chain}

The way of constructing ground states of the Majumdar-Ghosh chain with the Hamiltonian (1) suggests a generalisation of the argument for more general systems, discussed in Caspers (1982) and Caspers and Magnus (1983b). All these systems have the property that the SP states are stationary states, and for special conditions they are ground states. It remains an open question whether or not they are the only ground states under these special conditions. A crucial step in the argument in $\$ 2$ of this paper is the conclusion that all groups of three consecutive spins are in a doublet state: this is a necessary and sufficient condition for the two sPS to be the only possible ground states. Again in Caspers (1982) and Caspers and Magnus (1983b) the total Hamiltonian is subdivided into terms corresponding with a small group of spins: whereas in the simple MG chain we only have groups of three consecutive spins, we now have a subdivision corresponding with $3,5, \ldots, 2 l_{M}+1$ spins, $2 l_{M}$ being the range of the interaction ( $l_{\mathrm{M}}=1$ for the simple MG chain). The coupling between a spin pair at positions $i$ and $i+n$ is now given by

$$
\begin{aligned}
& 4 \sum_{r=x, y, z} J_{r} \gamma(n) S_{i}^{r} S_{i+n ;}^{r} \quad \gamma(n)=\sum_{l=1}^{l_{m}}(2 l-n+1) \delta^{(l)}, \\
& l_{n}=\frac{1}{4}\left[2 n+1-(-)^{n}\right],
\end{aligned}
$$

this coupling being anisotropic generally and having a more complicated dependence on $n$. For the case that $\left\{J_{x}, J_{y}, J_{z} \geqslant 0, J_{x}+J_{y}>0, J_{y}+J_{z}>0 ; \delta^{(l)} \geqslant 0, l=2, \ldots, l_{\mathrm{M}} ; l^{(1)}>\right.$ $0\}$ one easily checks that all groups of three consecutive spins are in a doublet state and consequently the ground state is a sPS. We believe, however, that there exists a larger set of parameters for which this is true. So far we were not able to formulate these more general conditions on the parameter set.

\section{Discussion of the results}

Because of the fact that two ground states of the Majumdar-Ghosh chain are exactly known, this system is interesting as a model for antiferromagnetic ordering. Recently a lot of effort has been put into the determination of the elementary excitations. These excitations seem to have a character that differs in an essential way from the well known 
spin waves in the nearest-neighbour Heisenberg chain (cf Des Cloizeaux and Pearson (1962) and Johnson and McCoy (1972)).

In this paper we have proved that the two ground states already known in the literature are the only ones. We also tried to gain more insight into the nature of the elementary excitations and made it plausible that there exists a gap in the spectrum. This could be of interest in the study of realistic physical systems in which there exists a predominant interaction within chains, which is of the Majumdar-Ghosh type.

\section{Appendix}

The defect state $\| e\rangle_{j}$, corresponding with the index set $\{j-4, j-3, \ldots, j+3, j+4\}$ is given by

$$
\begin{aligned}
(1 /(4 \sqrt{3}))[-2 \mid & +-+++--+-\rangle+2|-++++---+\rangle+2|+---++++-\rangle \\
& -2|-+--+++-+\rangle+1|+++-+--+-\rangle+1|+-++++---\rangle \\
& -1|++-++---+\rangle-1|+++-+---+\rangle+1|+-+++-+--\rangle \\
& -1|-++++-+--\rangle+1|++-++--+-\rangle-1|-++++---\rangle \\
& +1|-+--+-+++\rangle+1|---++++-+\rangle-1|--+-++++-\rangle \\
& -1|---+++++-\rangle+1|-+--++-++\rangle+1|-++-+++--\rangle \\
& -1|-++-+--++\rangle-1|-+-++--++\rangle+1|-+-++++--\rangle \\
& -1|--++++--+\rangle+1 \mid--++++-+-)-1|--+++-+-+\rangle \\
& +1|--+++-++-\rangle+1|--+-+++-+\rangle-1|+---++-++\rangle \\
& -1|+---+-+++\rangle-1|+-+-+++--\rangle+1|+-+-+--++\rangle \\
& -1|++--+-++-\rangle+1|++--+-+-+\rangle-1|++--++-+-\rangle \\
& +1|++--++--+\rangle+1|+--++--++\rangle-1 \mid+--++++--)]
\end{aligned}
$$

\section{References}

Bader H P and Schilling R 1979 Phys. Rev. B 193556

Caspers W J 1982 Physica 115A 275

Caspers W J and Magnus W 1982 Phys. Lett. 88A 103

1983a J. Phys. C: Solid State Phys. 166395

1983b Physica 119A 29 !

Des Cloizeaux J and Pearson J J 1962 Phys. Rev. 1282131

Johnson J D and McCoy B M 1972 Phys. Rev. A 61613

Klein D J 1982 J. Phys. A: Math. Gen. 15661

Majumdar C K 1970 J. Phys. C: Solid State Phys. 3911

Majumdar C K and Ghosh D K 1969a J. Math. Phys. 101388 1969b J. Math. Phys. 101399

Majumdar C K, Krishan K and Mubayi V 1972 J. Phys. C: Solid State Phys. 52896 Shastry B S and Sutherland B 1981 Phys. Rev. Lett. 47964

van den Broek P M 1980 Phys. Lett. 77A 261 